\title{
Does Quantum Mechanics Predict the Existence of Soul?
}

\author{
Hassan H.Mohammed
}

\section{ABSTRACT}

A person may possess a body-soul duality that is an extension of the waveparticle duality of subatomic particles. Both dualities participate in the element of subjectivism in explaining reality. This element of reality represents the philosophical aspect but has been neglected by contemporary cosmologists, who focus on the results of observation. In this paper, I demonstrate the scientific evidences for body-soul duality and cite the recent discoveries in quantum mechanics and quantum information and conclude that the metaphysics of this duality can be derived from the laws of nature.

Keywords: Body-Soul, Consciousness, Contemporary cosmology, Dualism, Quantum field, Quantum information, Time reversal.
Published Online: December 24,2019

DOI : $10.24018 / 2019.1 .1 .3$

\section{Hassan H.Mohammed*}

Department of Physics, College of Science, Basrah University, and at Iraq University College, Department of Computer Technical Engineering, Al- Estiqlal- Street, Basrah, Iraq. (e-mail: dr.hassanh50@gmail.com)

*Corresponding Author

\section{INTRODUCTION}

Ancient philosophical theories of soul demonstrated by Plato and Aristotle's trace a development towards comprehensive articulation of a very broad conception of soul, that does not only include mental or psychological functions like thought, perception and desire, but also accounts for all the vital functions that any living organism performs [1]. By contrast, the theories of the Hellenistic period are interested more narrowly in the soul as something that is responsible for mental and psychological functions. In view of both opinions it can be emphasized the connection between soul and life in all of its functions and aspects. The term body usually refers to matter. The bodysoul dualism appeared in the ancient Greek and medieval philosophy; in modern era the philosopher René Descartes (1596-1650) [2] can be considered as the true founder of the dualistic doctrine. He considered the function of mind is thinking and completely different from that of the body, that has dimensions; for him each could exist apart from the other. Furthermore, the existence is confined into two substances: the extended and the mental things. He ruled out the interaction between the matter and the soul.

Macmurray [3] has discussed the validity of the mindmatter dualism as inevitable thus, he qualified the philosophy of Descartes about this subject. He concluded that all the outstanding philosophical systems have been either monistic or plurastic.

Spinoza [4] thought God is a unique substance in the universe. He presented his picture of God as is the infinite, necessarily existing (that is uncaused). He considered God to be the creator of the world in such a way that a perfect harmony exists between mental and physical events. Søren
Kierkegaard [5] Danish philosopher (1813-1855) had an opinion that human existence is two- sided, one side is nature (in time), and the second is the soul (outside of time), and finds man partly in the space and time and partly in the immortal and infinity. His point of view about the duality body-soul approaches that of Spinoza by the existence of parallelism between the body and the soul and not interaction and decides the essential combination between their actions. The modern dualist Karl Popper [6] believes that there are three worlds: first, is the world of physical objects, second, is the states of consciousness, third, is the world of objective thought. The second world is the link between the first and the third ones. For him quantum mechanical action of the soul on matter is possibly regarded to be subtle.

Wigner [7] explored that the concept of consciousness is at the forefront of the researcher's attention. This is based on the fact that laws of quantum mechanics cannot be properly formulated without the reference to consciousness. This fact was embodied in the effect of the observer's awareness of the characteristics of the physical objects under experiment.

In his review paper of Jeffrey, Barrett [8] concluded that the static collapse caused by minds affect the quantummechanical states of physical systems and the objective, observable properties of the cosmological world as well.

Many scientific researches directed at the relationship between body and soul have been conducted using quantum field theories. However, Bohm first proposed the basic notion of the relationship between mind and matter is participation rather than interaction [9]. This proposal was based on the assumption that a series of orders of superquantum potentials constitute active information that manifests itself in the movements of the particles; leading to 
the relationship of various levels of subtlety in mind (thought, feeling, etc.). Although this view seems reasonable in terms of physical processes, it lacks the relationship with molecular biology in the brain. Quantum computers have shown to have the ability to perform processes that conventional computers cannot, which reminds us of how our brains can achieve things that are still beyond artificial intelligence. Ball [10] has recently stated that it is difficult to ignore the link between consciousness and quantum mechanics. Penerose [11] first proposed the quantum effect feature on human cognition, and demonstrated that the structure involved in the quantum cognition might be protein strands called microtubules. These are found in most of our cells including the neurons in our brains. Penerose argued that vibrations of microtubules can adopt a quantum superpositions.

In spite of the numerous intensive researches that linked consciousness or soul to quantum theory, none of them has shown that the metaphysics is an element of reality.

There are extensive research areas that show the mechanisms of memory and consciousness in the brain. These researches have been conducted using quantum mechanics models and presented in many papers [12-14].

In conclusion, despite their importance these theoretical models and proposals still need experimental verifications to confirm the validity of quantum effects.

The basic concept of quantum mechanics is based on the hypothesis of particle-wave dualism. The particles are considered as a localized body, whereas the waves especially the electromagnetic ones can extend and be described as the energy dynamics. This problem was overcome by Louis de Broglie's (1887-1987) suggestion that a particle can be represented as a plane-wave which has a wavelength inversely proportional to its momentum. De Broglie's idea of matter wave was developed and hence wave equation of motion was derived consequently. This formalism gave correct values for hydrogen atom energies and more complicated atoms.

The description of both dualities, body-soul, and particle-wave demonstrated that the concept of one is complementary with the other.

Despite the fact that the scientific researches carried out in well-established institutions have recently stated to have a link between the body-soul and its particle-wave counterpart in quantum mechanics, they could not support the metaphysical dimension of reality.

\section{The SCIENTIFIC ARgumENT}

The scientific evidences on the dual body-soul and its relevance on the metaphysical dimension of reality can be described as follows:

A. The conception of time can be considered as one of the most pressing debate in contemporary cosmology.

Recently, scientists from Russia, America, and Switzerland have demonstrated the possibility of reversing the time arrow using a quantum algorithm on an IBM quantum computer [15]. This time reversal is a law of nature and it means that we remember the future not the past, and explain the processes in future to make predictions about the past. Since the future cannot be explained by reference to physical events, but rather it is attributed to metaphysical events. This indicated that the backward time dynamics gives an evidence that the metaphysics is a part of physical reality. The time reversal phenomenon contradicts the second law of thermodynamics, which states that the total entropy of an isolated system can never decrease over time. Also it contradicts the argument of Kant about the time as mere sensible and a priori element of reality. But at this point the situation changed through quantum theory and therefore we may not compare Kant philosophical system with our present situation in modern physics. However, the inverse of time concepts also appeared in the intensive work in quantum mechanics using weak measurements and corresponding weak values of quantum particles [16]. This technique of weak measurements of quantum particles carries profound implications of our understanding of reality, and their measurements are influenced by both the initial preselected quantum state (the past) and the final postselected quantum states (the future). The time evolution of the quantum particles flows both ways: the future influences the present. Since the future can only represent metaphysical realism, these recent research trends support the conclusion that the consciousness or soul can be considered as part of realism, and consciousness is associated with matter .

Many cosmologists, philosophers and thinkers discussed the problem of time. However, Barbour [17] argues that the concept of time does not exist and has no place in physics. For him, intervals of time do not pre-exist but are created by what the universe does. Unlike Barbour, Smolin's [18] argues that time must exist and that embracing its reality, which is the key to solving persistent problems in cosmology. He gives us a sense that reality is moving from the past through the present to the future, as not merely a feature of human psychology but is fundamental of physical reality. This idea is in contradiction to recent experimental results of time reversal as mentioned earlier. Marcheisini [19] evaluated the concepts of both contemporary cosmologists and concluded that without the aid of philosophy, and in particular the concept of metaphysics, cosmology is likely to keep running into dead ends. This evaluation reinforces our interpretation in terms of matter-soul duality, which contains non-physical element of reality. This element appeared in quantum mechanics when the particles found not to obey the known laws of classical physics. However, the concepts of time and space were put forward by Revolli, one of the contemporary cosmologist and one of the founders of loop quantum gravity [20], focusing his idea on events at which something might happen, rather than particles or fields. He considers events to be the basic constituents of universe. These concepts are consistent with that of Barbour from the point of view that the task of physics is to describe the relationships between events and time and space that are only manifested out of their interactions and the web causality between them. The fact that the theory of Revolli maintains that the 
difference between the past and the future is illusionary does not change the fact that we perceive a significant difference between them. Furthermore, the loop quantum gravity providing equations with which it explores the cosmological big bang. Martin Bojowald [21] reports in this issue on the possibility that we might able to deduce features of the other side of the bounce (before the big bang) by means of measurements performed in our side. He claimed that the analysis of pre-big bang era is practically impossible and cannot be fully justified within science itself. The full justification can be described by the combination of metaphysical event with the scientific one. The latter has been developed by Bojowald model of the cosmological quantum gravity. This means that the consciousness factor does not appear in his postulates of time and space. Bergson offered a deep understanding of time than that mentioned by contemporary cosmologists [19]. This constitute the accounts of the duration and evolutions as intrinsically creative processes; and a true rebirth of time, which is the intrinsic feature of the universe. The clearest examination of the matter consciousness was developed by Max Tegmark [22] who bridged the gap between the cosmologists and philosophers by introducing the idea that consciousness could be a state of matter " perceptronium". He proposed that there is a state of matter- just like solid, liquid or gas in which atoms are arranged into process information and give rise to subjectivity and consciousness. Just as there are many types of liquids, there are many types of consciousness. Tegmark argued that consciousness is relevant in solving an open problem at the very heart of quantum mechanics: the quantum factorization problem. The nature and role of the observer (consciousness) are found to be related to the tensor factorization matrices which play a central role.

B. Another dimension of the body-soul duality is related to the quantum information. Quantum information laws form a consistency network that creates all fields, particles, space-time and leads to a conclusive proof of the aspect of consciousness as an element of reality. John Wheeler [23] suggested the term " It from Bit" to symbolize the idea that every item of the physical world has at the bottom an immaterial source and explanation; that what we call reality arises in the last analysis from posing of yes-no questions and registering of equipment-evoked responses. In short, all physical things are information-theoretic in origin and this is a participatory universe [23]. Wheeler's suggestion of "It from Bit" does not devoid of all trace of information related to consciousness. The role of consciousness is coming from observer-participant by his choice of question, he decides about what feature of object he shall have the right to make a clear statement. However, understanding quantum states as an information system, exposes the energyinformation duality. The conservation of energy in the matter or physical world is called information conservation in the quantum world. As the measure of information is a dimensionless number, there is no longer mass or energy but an event or life. The fundamental equations of the theory of loop quantum gravity developed by Carlo Rovelli [24] have no explicit space or time variables. This means that the quantum field does not live in space and time. The space and time are almost properties of the material whose disappearance can be explained by the existence of metaphysical or spiritual characteristics. It seems that the subjective experiences take place in 'psycho-spacetime'. This formalism reinforces the interpretation of the quantum field in terms of the metaphysical nature of reality. In fact, Rovelli [25] in his recent book, the order of time, claimed that " the mystery of time is ultimately, perhaps, more about ourselves than about the cosmos; and in the first and greatest of all detective novels, Sophocles Oedipus Rex, the culprit turns out to be the detective. Alfred North Whitehead (1861-1947) [26], pictured the world as a process of events similar to Rovelli's concept, but unlike Rovelli, Whitehead explained that nature has the duality of mental and physical aspects. The quantum information leads again to the conclusion that the interference of cosmology and philosophy might give the complete interpretation of the events occurring in our universe. Furthermore, Gao Shan [27] demonstrated that consciousness is not reducible or emergent, but new fundamental property of matter. This provides a quantum basis for panpscychism. He argued that the conscious process is one kind of quantum computation process. Carlo Eduardo Maldonado [28] studied the relationship between quantum physics and consciousness and argued in favor of panpscychism. Thus, consciousness is innate to the universe. He demonstrated that there are levels of reality and hence also levels of consciousness. These various levels are different forms of processing information. Furthermore, consciousness and reality are closely entangled and the processing of information is the way in which the entanglement takes place.

C. The third dimension of the problem of consciousness in quantum mechanics is related to the interpretation of the wave function collapse when making measurements in microscopic scale phenomenon in physics. This phenomenon refers to the subjectivism of particle wave duality in quantum mechanics similar to the body- soul dualism in philosophy. Initially, the wave function is in superposition of position, and after measurements, enters a new state corresponding to the measurement result. When making measurement a definite position (eigenstate) is observed. This process is often called a wave function collapse. Collapses happen in reality, triggered by measurement events. One needs to specify the notion of measurement and clarify the basic principles. In this framework, collapse might underlie processes that are non-computable (mental, thought and understanding) [29]; therefore these processes might be important for consciousness. The entanglement between electrons on protein polymer called microtubles in the brain would be at the root of the conscious process. The microtubles could support the macroscopic quantum features known as BoseEinstein condensates. These condensates could be linked with other neurons via gap junctions. This 
permits quantum coherence to extend over a large area of the brain. When one of the areas of quantum coherence collapses, there is an instance of consciousness, and the brain has access to noncomputational process. The second interpretation of the wave function collapse is in relevance to cosmology. The wave function collapse can be explained in terms of several world interpretation; this means that a quantum event occurs anywhere in the universe replicates into its opposite in another universe [30]. This approach is the " multiple world hypothesis" of Everett [31] in which each possibility in a superposition evolves to form its own universe, resulting in an infinite multitude of coexisting 'parallel' worlds. The consciousness of the observer is supposed to 'split', so that there is one in each of the worlds for which the observer remains alive and conscious [10]. At present, even if this theory has not been found to be complete, but one thing is certain, i.e the collapse process of wave function is one kind of dynamical process, and it takes a finite time interval between the collapse time $t_{C}$ and the normal conscious time $t_{P}$ and $t_{C}>t_{P}$ was assumed [27]. Shan has demonstrated, the conscious being or the matter with consciousness can recognize the nonorthogonal states, while the usual physical measuring device or matter without consciousness cannot. He concluded that this distinguishability reveals a quantum effect of consciousness. This scenario leads to the conclusion that the aspect of the wave function collapse cannot be interpreted in terms of matter realism, but almost in metaphysical aspect of reality.

D. The most promising theoretical way of interpreting the body-soul duality and looking at the neural correlates of consciousness is the use of the framework of stochastic electrodynamics (SED). This framework is ideally suited for establishing the fundamental description level at which the physical and mental aspects of our universe can be merged into a coherent whole. This is based on the hypothesis that a deep understanding of consciousness is closely connected with a deep understanding of matter. In this manner, the present approach paves the way to complete theory of body-soul duality. While the conventional quantum field theory QFT, purely describes the quantum system, SED is capable of unveiling the mechanisms that account for the quantum behavior of matter [32]. In addition, this approach leads to the satisfying explanation of neural correlates consciousness as we will see later. According to SED all conscious processes are woven into the frequency spectrum of universal background field, called zero-point field ( $\mathrm{ZPF}$ ); and the electrically charged components of every physical system interact unavoidably with radiative background. The energy exchange between the constituents and the ZPF can reach equilibrium where the average power absorbed by the system compensates exactly the average radiative power. The balance situations are characterized by quantization conditions and corresponds to the stationary states predicted by the quantum theory [33]. Hence, any dynamical system in equilibrium with ZPF displays quantum behavior and falls into an attractor; thus acquiring a stochastic motion and behaving as a stochastic oscillator [34]. Each individual system responds to a specific set of relevant field modes that are selectively extracted from the full frequency spectrum of the ZPF. These frequency modes are involved in the maintenance of the equilibrium and become highly correlated, resulting in a partial organization of the local field [35]. Under phaselocked situation, this organization gives rise to collective cooperation and long range coherence. The pattern of phase-locked ZPF modes can be interpreted as information states, in an information space, which can be referred to as ZPF information states. This means the formation of an attractor imprints an information state on the ZPF with the information contents depending on the dynamical properties of the attractor. Each quantum system distinguishes itself by its specific set of resonance frequencies and the respective pattern of coupled ZPF modes. As a consequence, different attractors go along with different ZPF information states, and every information state is associated with a conscious state. This hypothesis reflects the dual nature of the ZPF as the carrier of both energy or matter and consciousness or soul, and expresses the pattern of phase-locked ZPF modes which determine both physical and the phenomenological properties of quantum system [36]. Further, the dynamical variability of a system determines the accessible spectrum of conscious states. This variability is characterized by the complexity of the attractor landscape, which defines both the degree of consciousness and the diversity of conscious experience of system. At this point, it is important to recall that the properties of quantum system are not intrinsic properties, but dynamically acquired properties that can be attributed to the system over the lifetime of an attractor. From practical point of view, Information in our central nervous system (CNS) is phase-locked, which allows information exchange between the brain in the matter world and the ZPF of mind in the quantum world. The download of human mind into a far unobservable quantum field could explain the metaphysical nature of events. This is confirmed by the fact that the living body exchanges information with the soul, and we conclude the existence of both physical and metaphysical processes. The physical brain has some mind (brain's software) before interacting with its ZPF. The living brain creates more mind upon interaction with the ZPF and creates new memory. Unlike the computer, nobody from outside assigns or can assign meaning to 
newly created neuron pattern but the living brain does by itself [37].

E. The quantum field approach developed by Bohm [9], leads to the following notes:

i- The relationship between mind or consciousness and matter is participation rather than interaction. The transfer of information between them is through perception [5].

ii- The quantum potential constitutes the active information to be uploaded to the computer memory, the soul quantum field, or the data conserves on the computer memory.

iii- Extending this view, each human being, similarly participate in an inseparable way in society, and in the planet as a whole, but in a greater collective mind.

F. The practical evidences for the existence of consciousness or soul can be:

i- Neural correlates of consciousness (NCC) experiment

The realization of the SED mechanism in the brain consists of distilling the neural correlates of consciousness (NCC). This proposal of NCC has been receiving greatest support according to which conscious states are associated with transient synchronization of neural activity in the local beta and gamma frequency band in the electroencephalographic (EEG). This relation between long distance synchronization and consciousness was experimentally verified by Melloni et al [38] and Gaillard et al [39]. This experiment was devoted to the comparison between conscious and nonconscious processing of briefly flashed words using a visual masking procedure while recording intracranial electroencephalogram (iEEG) in many subjects. It was found for conscious processing a large increase in spectral power in the gamma band, increases in longdistance phase synchrony in the beta range. These results confirms the neural correlates of conscious access.

ii- Time - frequency analysis of the local field potential (LPF)

the characterizing bursting can be observed in the gamma-band of LEP data at each electrode along with simulation of synthetic data and a noise model. The particular measures to test for gamma bursts were the duration (time scale) and frequency of operationally defined short bursts of gamma activity above prespecified threshold [40]. A time frequency analysis revealed that the source of gamma-band peaks is of stochastic nature and the gamma activity is indistinguishable from filtered noise. The conclusion drawn by this experiment is that a realistic model of the brain should be viewed as a resonant stochastic oscillator and noise could play a fundamental role in the biological information processing. When the activity of the brain reaches a stable attractor, ZPF information state is generated and conscious experience arises. iii- Experimental demonstration of backward time dynamics for an electron

This experiment was carried out by implanting two-and three- qubit reversal procedures utilizing the public IBM quantum computer [15]. The experimental model is associated with a one dimensional particle scattering on a two level impurity (TLI). This scattering problem is modeled by the evolution of the qubit register, where qubit describes both the state of the TLI and the state of the scattered particles. The findings of the experiment suggest several directions for investigating time reversal and backward time flow in real quantum systems.

iv- The wave function collapse experiment

A double-slit optical system was used to test the possible role of consciousness in the collapse of the quantum wave function. The ratio of the interference pattern's double-slit spectral power to its single-slit spectral power was predicted to decrease when attention was focused on the double-slit as compared to away from it. Data collected by 137 people in six experiments, involving a total of 250 test sessions, indicated that the spectral ratio decreased. Other 250 control sessions conducted without observers tested hardware, software and analytical procedures for potential artifacts; it was found out that no evidence of procedural, or analytical artifacts that might have been responsible for these effects. The results appeared to be consistent with consciousnessrelated interpretation of the quantum measurement problem [41].

\section{CONCLUSION}

The scientific evidences in this paper leads to the conclusion that quantum mechanics predicts the existence of soul or consciousness. This conclusion has been based on objective matter processes, such as the neural processes in the brain and the concomitant subjective conscious experience. The reversal time in quantum information and the weak measurements in quantum mechanics lead to conclude that the metaphysics of the body-soul duality can be derived from the laws of nature and the physics and metaphysics could be conceived as aspects of the external reality of our universe and the internal reality of our mind. The SED approach paves the way towards a complete theory of body-soul duality. The subject of immortality of the soul and the existence of life after death is supported by divine religions. This subject requires evidences from a scientific point of view.

\section{ACKNOWLEDGMENT}

I would like to thank Professor Mohammed A. Habeeb at Al-Nahrain University, physics department for providing me with the recent books in quantum mechanics and quantum consciousness and for stimulating discussions. My thanks also to Dr. Walaa M.Jawad Saeed, post graduated from South California University for his support and encouragement. 


\section{REFERENCES}

[1] Ancient Theories of Soul, Stanford Encyclopedia of Philosophy, (2009). Available: http:// plato. Stanford. Edu/ index.html.

[2] W. E. Pickren, and A. Rutherford, A History of Modern Psychology in Context. A John Wiley \& Sons, INC, 2010.

[3] J. Macmurray, "The dualism of mind and matter". Philosophy, 10(39), pp. 264-278, 1935.

[4] B. Spinoza, Stanford Encyclopedia of Philosophy, (2016). Available: http:// plato.stanford.edu/entries/spinoza.

[5] A. L. Nielson, "Existential practice: relating to the infinite", Journal of Philosophy and Cultural Studies 1, pp. 68-77, 2014.

[6] H. Wehrli, Metaphysics Chirality as the Basic Principles of Physics. Copyright Hans Wehrli. 2008. www.hanswehrli.ch.

[7] E. Wigner, Remarks on the Mind-Body Question. Symmetries and Reflections. Cambridge (M.I.T. Press) 171-184, 1970.

[8] J.A. Barrett, "Quantum Mechanics and Dualism". U. Mzixner \& A. Corradini, Eds, 2014, pp. 65-82.

[9] D. Bohm. "A new theory of the relationship of mind and matter". Philosophical Psychology, 3(2), pp. 271- 286, 1990.

[10] P. Ball, "Why the many world interpretation has many problems". Quantum Physics, October 18, 2018.

[11] R. Penerose, The Emperor's New Mind, Oxford University Press, 1989.

[12] J. Sànchez-Cañizares, "The mind-brain problem and the measurement paradox of quantum mechanics: should we disentangle them?", NeuroQuantology, 12(1), pp. 76- 95, 2014.

[13] P. R. Blanquet, "New issue to modeling intentionality in the field of consciousness", Journal of Behavioral and Brain Science 5, pp. 348367,2015

[14] M. Bischof. and E. D. Giudice, "Communication and the emergence of collective behavior in living organisms. A quantum approach". Molecular Biology International Article ID987549, 19 Pages, 2013. Available: http://dx.doi.org/10.1155/2013/987549.

[15] G.B. Lesovik, I.A. Suslov, M.V. Sadovskyy, A.V. Lebedev, and V.M. Vinokur, "Arrow of time and its reversal on the IBM quantum computer". Scientific Reports 9:4396. 2019. Available: http://doi.org/10.1038/s41598.019.40765.6.

[16] Y. Aharonov, , E. Cohen, , M. Waegell and A. C. Elitzur, "The weak reality that makes quantum phenomena more natural: novel insights and experiments". Arxiv.org /ftp/ arxiv/ papers/1811/ 1811.04704. 2018.

[17] J. Barbour ,"The nature of time", arXiv: 0903.348901 [gr-qc] , 20 Mach, 2009.

[18] L. Smolin, Time Reborn: From the Crises in Physics to the Future of the Universe. Houghton Mifflin Harcourt, Boston. New York, 2013.

[19] P. Marchesini, "The end of time or time reborn? Henri Bergson and the metaphysics of time in contemporary cosmology", Philosophy and Cosmology, 21, pp. 140-152, 2018.

[20] C. Rovelli, "Forget time". arXiv:0903.3832v3 [gr-qc] 27 Mar. 2009.

[21] M. Bojowald. " What happened before the big bang?". Nature Physics, 3, pp. 523-525, 2007.

[22] M. Tegmark, "Consciousness as a state of matter", Chaos. Soliton \& Fractals 76, pp. 238-270, 2015. Available: http://dx.doi.org/10.1016/j.chaos.2015.03.014.

[23] J. A. Wheeler, "Information, physics, quantum: the search for links", Proc. $3^{\text {rd }}$ Int. Symposium Foundations of Quantum Mechanics. Tokyo, 354-368, 1989.

[24] C. Rovelli, "Physics needs philosophy, philosophy needs physics", Foundation of Physics. 48(5), pp. 481-491, 2018. Available: http://hal.archieves-overtes.fr/hal-01771743.

[25] C. Rovelli, The Order of Time. Riverhead Books, New York. 2018.

[26] M. D. Segall, Physics of the World - Soul: The Relevance of Alfred North Whitehead's Philosophy of Organism to Contemporary Scientific Cosmology. Third Edition, Oxford, UK. 2018.

[27] G. Shan, "Quantum consciousness and panpsychism A solution to the hard problem", NeuroQuantology, 4(2), 166-185, 2006.

[28] C. E. Maldonado, "Quantum physics and consciousness: A (strong) defense of panpscychism". Available: http://dx.doi.org/10.1590/01013173.2018.v41esp.07.p101. 2018.

[29] R. Penerose, and S. Hameroff, "Consciousness in the universe: neuroscience, quantum space-time geometry and Orch OR theory", Journal of Cosmology, Vol. 14, 2011.

[30] P. Ball, "Quantum Biology: An Introduction", A talk at the Royal Institution. London, January 28, 2015.

[31] H. Everett, " Relative state formulation of quantum mechanics". Rev. Mod. Phys., 29, pp. 454-462, 1957.

[32] J. Keppler, "The role of the brain in conscious processes: A new way of looking at the neural correlates of consciousness", Frontiers in Psychology, Vol. 9, 2018. doi: 10.3389/fpsyg.2018.01346.
[33] L. De la Peña, and A. M. Cetto,. "The foundations of linear stochastic electrodynamics". Found. Phys. 36, pp. 350- 368, 2006. doi: 10. 1007/s 10701-005-9020-1.

[34] L. De la Peña, and A. M. Cetto, "Quantum phenomena and the zero point radiation field II" . Found. Phys. 25, PP. 573- 604, 1995. doi: 10.1007/BF02059007.

[35] L. De la Peña, A. Valdés- Hernàndez, and A. M. Cetto, "Quantum mechanics as an emergent property of ergodic systems embedded in the zero-point radiation field". Found.Phys. 39, pp.1240-1272, 2009. doi: 10.1007/s10701-009-9348-z.

[36] J. Keppler, "A new perspective on the functioning of the brain and the mechanisms behind conscious processes", Frontiers in Psychology, Vol.4, 2013. doi: 10.3389/fpsyg.2013.00242.

[37] S. Hari, "Consciousness, mind and matter in Indian philosophy", Journal of Consciousness Exploration \& Research, 1(6), pp. 640-650, 2010.

[38] L. Milloni, C. P. Molina, M. D. Torres, W. Singer, and E. Rodriguez, "Synchronization of neural activity across cortical areas correlates with conscious perception". The Journal of Neuroscience, 14(11), pp. 2858-2865, 2007.

[39] R. Gaillard, S. A. Dehaene, S. H. D. Baulac, M.L. Cohen, and L. Naccache, "Converging intracranial markers of conscious access", PLoS BIOLOGY, 7(3), pp. 472-492, 2009.

[40] S. P. Burns, D. Xing. and R. M. Sharply, 2011. "Is gamma-band activity in the local field potential V1 cortex a "Clock" or filtered noise"?. The Journal of Neuroscience, 31(26), pp. 9658-9664, (2011). DOI:10.1523/JNEUROSCI.0660-11.2011.

[41] D. Radin, L. Michel, K. Galdamez, P. Wendland, and R. Rickenbach, "Consciousness and the double-slit interference pattern: six experiments". Physics Essays, 25(2), pp. 157-171, 2012.

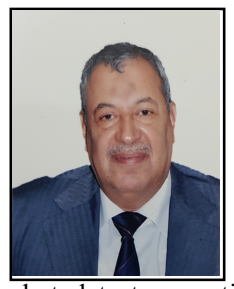

The author was born in Basrah city, the first of July 1951. He has a BSc in physics from Basrah University (honor) in 1972, MSc in (molecular physics) in 1975, DEA and $\mathrm{PhD}$ from the University of Paris 11 (Orsay), France in 1981. The thesis was in the field of (matrix isolation optical spectroscopy using laser sources). The fields of interests are: lasers in spectroscopy, lasers in medicine, nanotechnology, photodetectors, optical communications and tracking and philosophy of physics.

$\mathrm{He}$ worked as a lecturer and research staff at the University of technology and chief researchers and head of lasers and optoelectronics center at the Ministry of Science and Technology (MOST), Baghdad. In 2008 he joined the University of Basrah, physics department and worked as an assistant professor first and then as a full professor in physics. At present he connected with the Iraq University college, department of technical computer engineering as a lecturer and research staff.

Professor Mohammed has published 40 articles, supervised $36 \mathrm{MSc}$ and $\mathrm{PhD}$ theses during the research period, and also has 4 patents in applied physics field. He awarded the medal of scientests in Iraq for his contribution in scientific researches. 
